\title{
ADDITIVE GROUPS OF $T$-RINGS
}

\author{
GEORGE V. WILSON
}

\begin{abstract}
We build on a result of Bowshell and Schultz to give a complete characterization of the groups which occur as the additive groups of $T$-rings. This answers a question of Feigelstock.
\end{abstract}

In this paper, all groups are abelian. We write $R^{+}$for the additive group of a ring $R, t(G)$ for the torsion subgroup of a group $G$, and $G_{p}$ for the $p$-primary component of $G$.

Fuchs [F1, Problem 45] posed the problem of classifying those rings $R$ with the property that $R \approx \operatorname{End}_{\mathbf{z}}\left(R^{+}\right)$. Bowshell and Schultz $[\mathbf{B S}]$ found one class of rings with this property that they called $T$-rings. A unital ring $R$ is a $T$-ring if the map $m: R \otimes \mathbf{z} R \rightarrow R$ induced by multiplication $a \otimes b \rightarrow a b$ is an isomorphism. [BS] characterized $T$-rings in terms of their additive groups. In order to state their result, we recall some basic facts about torsion-free groups. A torsion-free group has rank one if and only if it is isomorphic to a subgroup of the rational numbers. Rank one groups are completely classified by an invariant called type. A rank one group is the additive group of a unital ring if and only if its type is idempotent under a natural product. Readers unfamiliar with this material are referred to [F2, $\S 85]$ for a thorough treatment.

We are now in a position to state the above-mentioned result.

PROPOSITION 1 [BS]. The following are equivalent for a unital ring $R$ :

(A) $R$ is a $T$-ring,

(B) (1) the quotient $R^{+} / t\left(R^{+}\right)$is a rank one group of idempotent type and

(2) if $R_{p}^{+}$is nonzero, it is cyclic and $R^{+} / t\left(R^{+}\right)$is p-divisible.

From this, one can easily see that a torsion group is the additive group of a $T$-ring if and only if it is cyclic; see [BS, 1.4]. This led Feigelstock to ask if the conditions in $B$ above are enough to guarantee that a nontorsion group is the additive group of a $T$-ring [ $\mathbf{F g}$, Question 4.7.30]. By Proposition 1, it suffices to determine if such a group is the additive group of some unital ring. In fact, these conditions are not sufficient and we now determine the minor additional restriction needed to give a $T$-ring.

PROPOSITION 2. Let $G$ be an abelian group which satisfies the conditions in (B) above. Then either $G \approx t(G) \oplus G / t(G)$ or else $\bigoplus G_{p} \leq G \leq \prod G_{p}$.

PROOF. Since we assume that each nonzero $p$-component $G_{p}$ is cyclic, the $G_{p}$ are pure and bounded, hence they are summands $\left[\right.$ F2, 27.5]. Let $\pi_{p}: G \rightarrow G_{p}$ be a projection map and let $\alpha=\prod \pi_{p}: G \rightarrow \prod G_{p}$ be the product of these maps. Suppose that there is even one element $x$ with infinitely many of the $\pi_{p} x \neq 0$. In

Received by the editors September 30, 1985 and, in revised form, December 27, 1985.

1980 Mathematics Subject Classification (1985 Revision). Primary 20 K99. 
this case, we claim that $\alpha$ is an injection. Let $q: G \rightarrow G / t(G)$ be the quotient map, $q(x) \neq 0$. Take any $g \in G$. If $g \in t(G)$, then of course $\alpha(g) \neq 0$. Next suppose $g \notin t(G)$. Since $G / t(G)$ is assumed to be a rank one group, $q(g)$ is a rational multiple of $q(x)$, i.e. $m q(x)=n q(g)$ for some integers $m$ and $n$. This implies that $m x=n g+t$ for some $t \in t(G)$. Since $x$ has infinitely many nonzero projections $\pi_{p} x$, so does $m x$. Since $t$ has only finitely many nonzero projections, $n g$ must have infinitely many and so $g$ does also. Thus, $\alpha g \neq 0$ and $\alpha$ is an injection.

Next suppose that there is no element with infinitely many projections. Then every element $g \in G$ can be written $g=k+t$, where $k \in \operatorname{ker} \alpha$ and $t \in t(G)$. Since $\operatorname{ker} \alpha \cap t(G)=0, G=\operatorname{ker} \alpha \oplus t(G)$.

We can now complete the picture of the additive groups of $T$-rings.

Proposition 3. A group $G$ is the additive group of a $T$-ring if and only if

(1) it satisfies the conditions of (B) in Proposition 1 and

(2) there is some $g \in G$ such that for every prime $p, \pi_{p} g$ generates $G_{p}$.

Proof. Say $G \approx R^{+}$for a $T$-ring $R$. Proposition 1 shows that (B) holds. It is easy to see that for $1 \in R, \pi_{p} 1$ generates $G_{p}$ for every $p$.

Conversely, assume that $G$ satisfies (1) and (2). By Proposition $2, G \approx t(G) \oplus$ $G / t(G)$ or $\bigoplus G_{p} \leq G \leq \prod G_{p}$. Suppose that $G \approx t(G) \oplus X$, where $X$ is a rank one, torsion-free group of idempotent type. Write the $g$ given in condition (2) as $g=t+x, t \in(G), x \in X$. Since $\pi_{p}(x)=0$ for all $p$ and $\pi_{p}(t) \neq 0$ for only finitely many $p, \pi_{p}(g)$ is nonzero for only finitely many $p$. Since $\pi_{p}(g)$ generates each $G_{p}$, only finitely many $G_{p}$ are nonzero. Since each $G_{p}$ is cyclic, $t(G)$ is cyclic and carries a unital ring structure. Since $X$ is rank one of idempotent type, it also has unital ring structure. Clearly, $G \approx t(G) \oplus X$ carries the product ring structure.

Suppose $\bigoplus G_{p} \leq G \leq \prod G_{p}$. Let $q: G \rightarrow G / t(G)$ be the quotient map. Choose $g \in G$ such that for all $p, \pi_{p} g$ generates $G_{p}$ and so that $q(g)$ has an idempotent height sequence. Give each $G_{p}$ the ring structure that makes $\pi_{p} g$ the unity of $G_{p}$ and give $\prod G_{p}$ the product ring structure. We claim that $G$ is a subring. Clearly, if $t \in \bigoplus G_{p}$ and $x$ is any element of $\prod G_{p}$, then $x t \in \bigoplus G_{p} \leq G$, so we must show that the product of nontorsion elements of $G$ is again in $G$. Take $x, y \in G$ and write $j x=m g+t, k y=n g+s$ with $j, k, m, n \in \mathbf{Z}, t, s \in t(G)$. Since $q(g)$ has idempotent type and is divisible by $j$ and $k$, it is divisible by $j k$. Choose $z \in G$ with $j k z=m n g+u, u \in t(G)$. With this choice, $j k(z-x y) \in t(G)$, so $v=z-x y \in t(G)$ and $x y=z-v \in G$. We see that $G$ is a unital subring of $\prod G_{p}$ and so is a $T$-ring.

It is fairly easy to see that any group satisfying the conditions of (B) has a ring structure which makes the multiplication map $G \otimes G \rightarrow G$ an isomorphism. The extra condition in Proposition 3 is simply to insure that this is a unital ring structure.

\section{REFERENCES}

[BS] R. A. Bowshell and P. Schultz, Unital rings whose additive endomorphisms commute, Math. Ann. 228 (1977), 197-214.

[F1] L. Fuchs, Abelian groups, Publ. House Hungarian Acad. Sci., Budapest, 1958.

[F2] _ Infinite Abelian groups, Vols. 1 and 2, Academic Press, New York and London, 1970 and 1973.

[Fg] S. Feigelstock, Additive groups of rings, Pitman, London, 1983.

Department of Mathematics, University of Georgia, Athens, Georgia 30602 